\title{
Green Synthesis of Barium Sulfate Particles Using Plant Extracts
}

\author{
Long $\mathrm{CHEN}^{1, *}$, Jianli WANG ${ }^{2}$, Hongfeng $\mathrm{WANG}^{2}$, Yuchuan $\mathrm{ZHENG}^{1}$, Zhaopeng \\ $\mathrm{QI}^{1}$, Guangru CHANG ${ }^{1}$, Shaohua XU ${ }^{1}$, Ruifeng $\mathrm{LI}^{1}$, Tiantian $\mathrm{WU}^{1}$, Wen $\mathrm{XU}^{1}$ \\ ${ }^{1}$ School of Chemistry and Chemical Engineering, Huangshan University, Anhui, Huangshan, 245041 \\ ${ }^{2}$ School of Mechanical and Electrical Engineering, Huangshan University, Anhui, Huangshan,
}

245041

\begin{abstract}
The biological molecules in the extracts of four fruits or vegetables: kiwifruit, oranges, tomato and carrot, were used as templates to synthesize barium sulfate $\left(\mathrm{BaSO}_{4}\right)$ particles. The products were characterized by scanning electron microscopy, Fourier transform infrared spectroscopy and X-ray power diffractometry. The results showed that, leaf-shaped barite $\mathrm{BaSO}_{4}$ crystals with toothed edge were obtained with kiwifruit extracts; thorn spherical barium sulfate crystals with diameter of 2-4 micrometers were produced with tomato extracts; rod-like or quasi-spherical $\mathrm{BaSO}_{4}$ crystals with size of several hundred nanometers to several micrometers were gained with orange extracts; while quasi-spherical $\mathrm{BaSO}_{4}$ nano-crystals were obtained with carrot extracts. The formation mechanism of $\mathrm{BaSO}_{4}$ is also discussed, showing that the proteins, carbohydrates, vitamins and organic acids in above four kinds of fruits or vegetables may provide nucleation sites, controlling the growth of $\mathrm{BaSO}_{4}$ crystals with different morphologies.
\end{abstract}

\section{Introduction}

Barium sulphate $\left(\mathrm{BaSO}_{4}\right)$ is generally white or colorless, chemically inert, insoluble in water, with high density, and the main source of barium[1]. It is widely used as a shading material for the x-ray photography, a gammaray absorber, a white pigment and etc because it is non-harmful to humans. $\mathrm{BaSO}_{4}$ is also one of the most important fillers used in the plastics, rubber and paint industries, and is also used in pharmaceutical formulations[2]. It can be used for X-ray attenuation instead of shielding made from lead when incorporated into polymeric materials to form composite [3].

* Corresponding author:chenlong200808@163.com 
In recent years, more attention has been paid on synthesis of $\mathrm{BaSO}_{4}$ nanoparticles for their multiple applications in the oil industry, electronics, TV screen, glass, car filters, paint industry, ceramics and medicine, etc. Many approaches have been chosen to control the size and morphology of $\mathrm{BaSO}_{4}$ particles. These methods could be classified as direct precipitation, microemulsion, membrane separation and organic modification [1]. M. Ismaiel et al have synthesized $\mathrm{BaSO}_{4}$ nanoparticles by precipitation method using polycarboxylate as a modifier[4]. D. Adityawarman have obtained $\mathrm{BaSO}_{4}$ nanoparticles in a non-ionic microemulsion[5]. G. Wu et al have precipited $\mathrm{BaSO}_{4}$ nanoparticles through impinging streams[6]. A. Gupta have prepared $\mathrm{BaSO}_{4}$ nanoparticles using sodium hexa metaphosphate as a stabilizer[7]. Very recently, the synthesis of $\mathrm{BaSO}_{4}$ nanoparticles by a chemical precipitation route without polymer stabilizers has been reported[1].

In this paper, we have synthesized $\mathrm{BaSO}_{4}$ particles by a very simple and green method. The $\mathrm{BaSO}_{4}$ particles especially nanoparticles obtained may be used as filler in plastics. The possible formation mechanism of the $\mathrm{BaSO}_{4}$ particles directed by biomolecules of the fruits or vegetables including kiwifruit, oranges, tomato and carrot is discussed. This report may be very significant for biomineralization research and green synthesis of functional inorganic materials.

\section{Experimental}

\subsection{Materials and Instruments}

Barium chloride dihydrate $\left(\mathrm{BaCl}_{2} \cdot 2 \mathrm{H}_{2} \mathrm{O}\right)$, anhydrous sodium sulfate $\left(\mathrm{Na}_{2} \mathrm{SO}_{4}\right)$, and ethanol were purchased from reagent plant of Shanghai (Shanghai, China), and were analytically pure. They were used without further purification. Four kinds of fruits or vegetables: kiwifruit, oranges, tomato and carrot were purchased from a local farmer. Double-distilled water was used in all experiments.

The samples were characterized by Fourier transform infrared spectroscopy (Nicolet 870 , America) using $\mathrm{KBr}$ pellets over the wave number range of $400-4000 \mathrm{~cm}^{-1}$ with a resolution of $4 \mathrm{~cm}^{-1}$ and X-ray diffractometer (D8 ADVANCE, Germany) using CuKa radiation at a scan rate of $0.06^{\circ} 2 \theta \cdot \mathrm{S}^{-1}$. The morphologies of the samples were observed by scanning electron microscopy (SEM) (KYKY-EM3200, China) with an accelerating voltage of $20 \mathrm{kV}$.

\subsection{Methods}

First, $80 \mathrm{~g}$ of each fruit or vegetable were washed carefully, and then crushed to extract juice, and the juice was mixed proper amount of double-distilled water, obtaining $90 \mathrm{~mL}$ of the fruit or vegetable extracts aqueous solution. After that, $20 \mathrm{~mL}$ of each solution was mixed with $40 \mathrm{~mL}$ of $0.05 \mathrm{~mol} / \mathrm{L} \mathrm{BaCl}_{2}$, completely, obtaining $60 \mathrm{~mL}$ of mixed solution 
containing fruit or vegetable extracts and $0.0333 \mathrm{~mol} / \mathrm{L} \mathrm{BaCl}{ }_{2}$. Then each of the mixed solution was blended with $40 \mathrm{~mL}$ of $0.05 \mathrm{~mol} / \mathrm{L} \mathrm{Na}_{2} \mathrm{SO}_{4}$ very slowly. The white precipitates produced in the mixed systems. The control experiment was also performed without adding of fruit or vegetable extracts. The above five reaction systems were placed at room temperature for 24 hours.

The white precipitates produced in the reaction solutions were separated by centrifugation (centrifugating rate, $4000 \mathrm{rpm}$ ), washed three times with double-distilled water and ethanol, respectively, and then vacuum dried for further determination.

The sizes and morphologies of the precipitates were examined by scanning electron microscopy (SEM), while their crystal phases were determined by Fourier transform infrared spectroscopy (FT-IR) or X-ray powder diffractometry (XRD).

\section{Results and discussion}

Fig.1 indicates SEM images of $\mathrm{BaSO}_{4}$ particles obtained in different systems. From Fig.1a and b, it can be seen that the particles obtained without any exracts are leaf-shaped with toothed edge. The size of each "leaf" is about 4-8 $\mu \mathrm{m}$. Fig. 1c and d shows that the particles obtained with kiwifruit extracts are also leaf-shaped, but the sizes of them become slightly smaller. When in the presence of tomato extracts, many thorn spheres with diameters of 2-4 $\mu \mathrm{m}$ are produced (Fig. 1e and f). While with orange extracts, quasi-spherical or rod-like $\mathrm{BaSO}_{4}$ particles with sizes of several hundred nanometers are gained (Fig. $1 \mathrm{~g}$ and h). At last, it can be seen from Fig. $1 \mathrm{i}$ and $\mathrm{j}$, uniform $\mathrm{BaSO}_{4}$ quasi-spheres with diameter of several hundred nanometers are obtained in the presence of carrot extracts .

From the results above, it can be concluded that the extracts of the fruits or vegetables have an influence on the morphologies of $\mathrm{BaSO}_{4}$ particles.

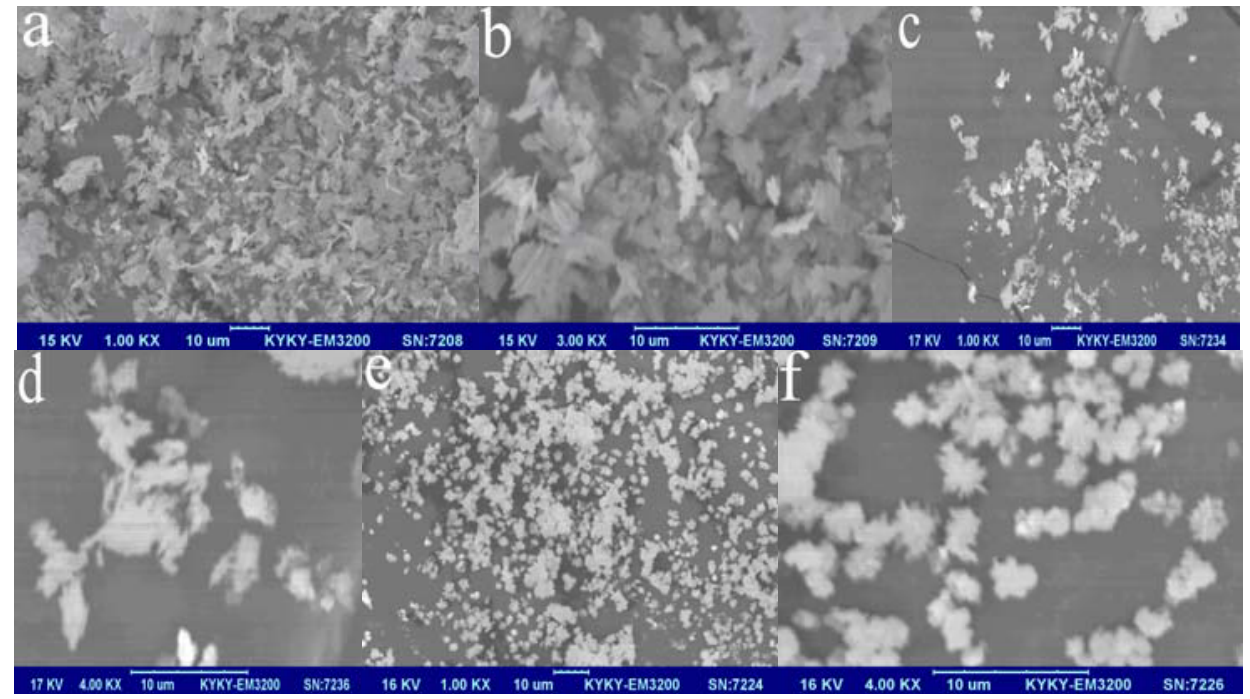




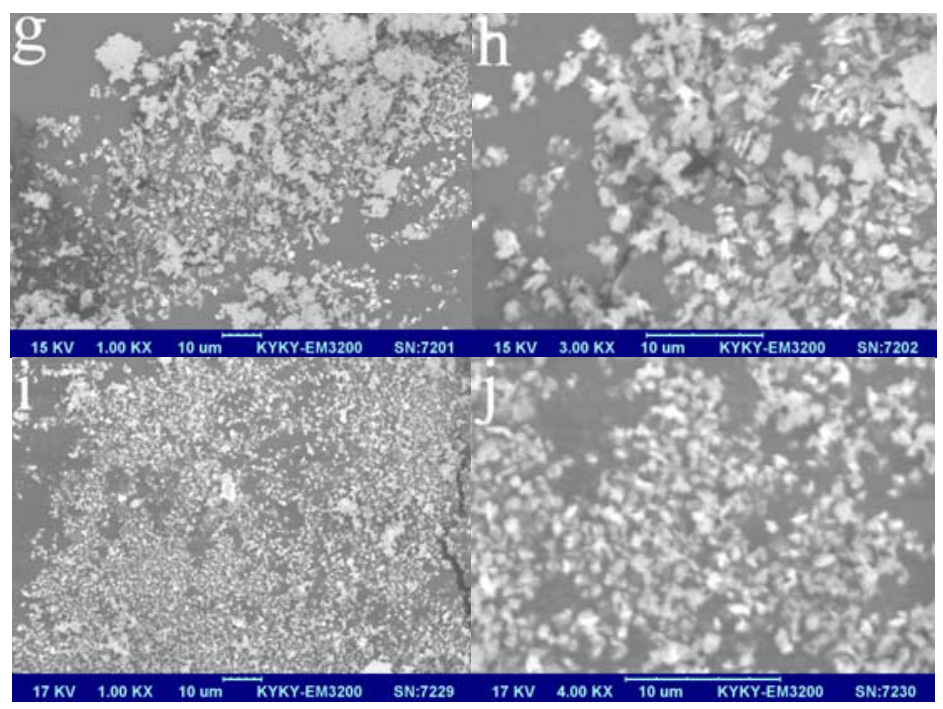

Fig. 1 SEM images of the products obtained in different systems.

(a, b: without any extracts; c, d: with kiwifruit extracts; e, f: with tomato extracts; g, h: with orange extracts; i, j:

with carrot extracts;

$\mathrm{b}, \mathrm{d}, \mathrm{f}, \mathrm{h}$ and $\mathrm{j}$ are the magnified images of a, c, e, g and i, respectively.)

In order to confirm the component of the products, FT-IR spectra of them obtained in different systems were measured. Fig. 2 shows the related FT-IR spectra, it can be seen that the characteristic peaks of the products are located at 1190-1070, 984 and $849 \mathrm{~cm}^{-1}$ assigned to out of plane bending vibrations of $\mathrm{SO}_{4}{ }^{2-}$. This suggests that the products obtained in different conditions are $\mathrm{BaSO}_{4}$ particles. The absorption peaks at about 1635 and $1550 \mathrm{~cm}^{-1}$ may be assigned to amide I $\left(v_{\mathrm{C}=\mathrm{O}}\right)$ and II $\left(\delta_{\mathrm{N}-\mathrm{H}}\right)$ absorptions of protein(s), respectively. This implies that the biomolecules such as proteins in the extracts of the fruits or vagetables might finally exist in the products.

Fig. 3 shows XRD patterns of $\mathrm{BaSO}_{4}$ crystals obtained in different systems. From Fig. $3 \mathrm{a}$, it can be seen that the XRD pattern of the $\mathrm{BaSO}_{4}$ crystals obtained without any extracts displays the following diffraction peaks $\left(2 \theta\left[^{\circ}\right]\right): 19.95^{\circ}, 22.45^{\circ}, 22.80^{\circ}, 24.87^{\circ}, 25.85^{\circ}$, $26.84^{\circ}, 28.75^{\circ}, 31.53^{\circ}, 32.80^{\circ}$ and $42.60^{\circ}$, which can be correlated to the (hkl) indices (020), (101), (111), (200), (021), (210), (121), (211), (002) and (140), of barite (JCPDS card number 83-1718). This suggests that the products are $\mathrm{BaSO}_{4}$ crystals. Fig. 3b-e indicate almost the same XRD results, revealing that the products gained with the four kinds of extracts are both barite crystals.

If seen clearly, the intensity of crystal face (020) in Fig. 3a is stronger than that of it in Fig. 3b-e. It may be speculated that the extracts of the fruits or vegetables are able to inhibit the (020) face growth of $\mathrm{BaSO}_{4}$ crystals. 


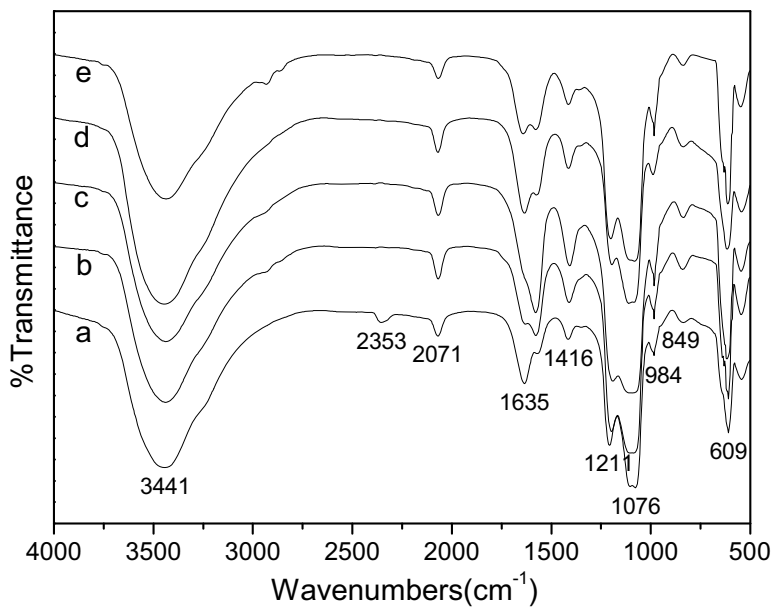

Fig. 2 FT-IR spectra of the products obtained in different systems.

(a: without any extracts; b: with kiwifruit extracts; c: with tomato extracts; d: with orange extracts; e: with carrot extracts)

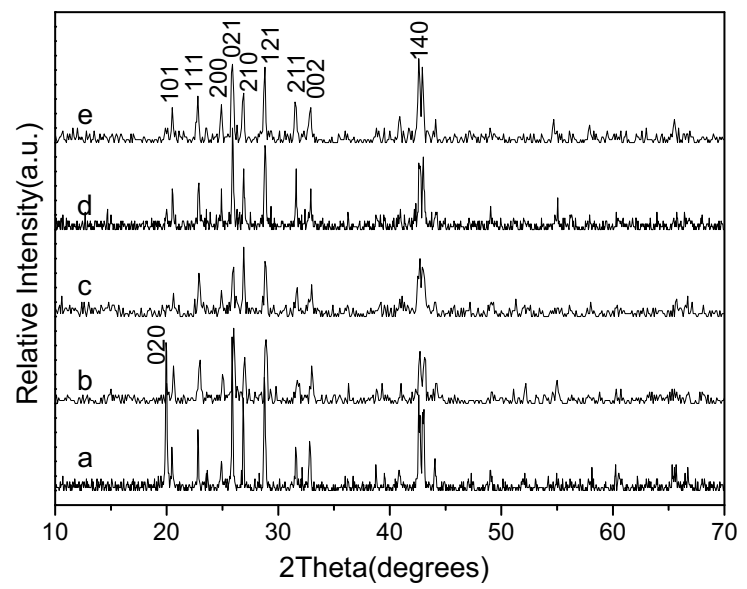

Fig. 3 XRD patterns of the products obtained in different systems.

(a: without any extracts; b: with kiwifruit extracts; c: with tomato extracts; d: with orange extracts; e: with carrot extracts)

The extracts of fruits and vagetables including kiwifruit, tomato, orange and carrot contain mang kinds of organic macromolecules such as proteins, carbohydrates, vitamins and some organic acids. In previous reports, it is accepted that the soluble biomacromolecules such as acidic proteins, glycoproteins and polysaccharides in biological systems act as nucleators, growth modifiers, and anchoring units in the mineral formation [8]. Here, we speculate that $\mathrm{Ba}^{2+}$ ions may complex with the soluble biomolecules in the fruits and vegetables and form stable aggregates in solution firstly. Then the entrapped $\mathrm{Ba}^{2+}$ ions react with $\mathrm{SO}_{4}{ }^{2-}$ ions forming $\mathrm{BaSO}_{4}$ nuclei. At last, biomolecule aggregates adsorb onto faces of $\mathrm{BaSO}_{4}$ crystals, controlling the formation of $\mathrm{BaSO}_{4}$ particles with different 
morphologies.

\section{Summary}

Green synthesis of $\mathrm{BaSO}_{4}$ particles using the extracts of four fruits or vegetables including kiwifruit, oranges, tomato and carrot was performed. leaf-shaped barite $\mathrm{BaSO}_{4}$ crystals with toothed edge were obtained in kiwifruit juice; thorn spherical barium sulfate crystals with diameter of 2-4 micrometers were produced in tomato juice; rod-like or quasi-spherical $\mathrm{BaSO}_{4}$ crystals with size of several hundred nanometers to several micrometers were gained in orange juice; while quasi-spherical $\mathrm{BaSO}_{4}$ nano-crystals were obtained in carrot juice. The formation mechanism of $\mathrm{BaSO}_{4}$ was also discussed, showing that proteins, carbohydrates, vitamins and organic acids in above four kinds of fruits or vegetables can provide nucleation sites, controlling the formation of $\mathrm{BaSO}_{4}$ particles with different morphologies.

\section{Acknowledgements}

This work is supported by National Science Foundation of China (21401065), the Projects of Anhui Provincial Education Department (KJ2016SD55, KJ2013B268, KJHS2015B14，KJ2015A259，KJ2016A679，KJ2016A681，KJHS2016B05), Anhui Provincial Natural Science Foundation (10040606Q58), the Key Project of Anhui Provincial Universities Excellent Talent Support Program (gxyqZD2016302), and the Projects of Huangshan University (2014xkj007, 2014xkj010, 2015xkjq007, 2015xkjq008, 2015xkjq009).

\section{References}

1. B. Sifontes, E. Cañizales, J. Toro-Mendoza,E. Ávila, P. Hernández, B. A. Delgado, G. B. Gutiérrez, Y. Díaz, E. Cruz-Barrios, Obtaining highly crystalline barium sulphate nanoparticles via chemical precipitation and quenching in absence of polymer stabilizers, Journal of Nanomaterials (2015) Article ID 510376.

2. H. C. Kim, Y. S. Chung, Preparation and radiopaque properties of chitosan/ $/ \mathrm{BaSO}_{4}$ composite fibers, Fibers and Polymers 14(2013) 292-297.

3. J. Unsworth, B. A. Lunn, P. C. Innis, M. Mapson, X-ray attenuation properties of electrically insulating barytes/epoxy composites. Journal of Materials Science Letters, 12(1993) 132-134.

4. M. Ismaiel, I. Mostafa, Synthesis of $\mathrm{BaSO}_{4}$ nanoparticles by precipitation method using polycarboxylate as a modifier, American Journal of Nanotechnology 2(2011) 106-111.

5. D. Adityawarman, A. Voigt, P. Veit, K. Sundmacher, Precipitation of $\mathrm{BaSO}_{4}$ nanoparticles in a non-ionic microemulsion: identification of suitable control 
parameters, Chemical Engineering Science 60(2005) 3373-3381.

6. G. Wu, H. Zhou, S. Zhu, Precipitation of barium sulfate nanoparticles via impinging streams, Materials Letters 61(2007) 168-170.

7. A. Gupta, P. Singh, C. Shivakumara, Synthesis of $\mathrm{BaSO}_{4}$ nanoparticles by precipitation method using sodium hexa metaphosphate as a stabilizer, Solid State Communications 150 (2010) 386-388.

8. A. Berman, J. Hanson, L. Leiserowitz, T. F. Koetzle, S. Weiner, L. Addadi, Biological control of crystal texture: a widespread strategy for adapting crystal properties to, Science 259 (1993) 776-779. 